\title{
Condições sociodemográficas e de saúde de idosos residentes em domicílio no município de Jequié-BA
}

\section{Socio-demographic and health status of elderly people living at home in Jequié} city, state of Bahia, Brazil

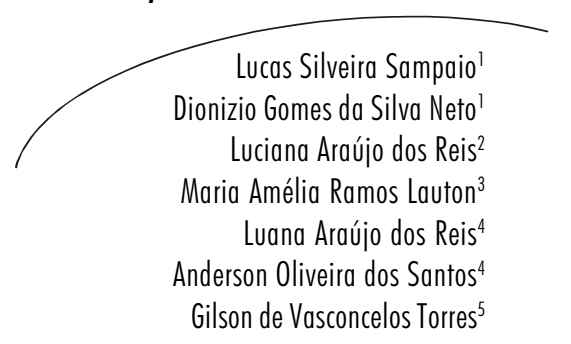

\section{Resumo}

O envelhecimento populacional é uma resposta à mudança de alguns indicadores de saúde, especialmente a queda da fecundidade e da mortalidade e o aumento da expectativa de vida. O crescimento elevado da população idosa brasileira é uma questão de saúde pública, e vem acentuando-se muito nas últimas décadas. Deste modo, este estudo objetiva conhecer as condições de saúde e sociodemográficas de idosos residentes em domicílio no município de Jequié-BA. É uma pesquisa de caráter exploratório descritivo com delineamento transversal e abordagem quantitativa. Identificou-se que $51,7 \%$ dos idosos eram do sexo masculino, $37,8 \%$ possuíam idade entre a faixa etária de 70 a 79 anos e $96,5 \%$ não eram alfabetizados; e a patologia mais encontrada entre os idosos foi a hipertensão, com 73,3\% dos acometimentos, seguida do diabetes, com $17,2 \%$. O estudo reforça a importância do planejamento de políticas públicas direcionadas às necessidades específicas da população idosa, além de outros estudos que possibilitem ampliar o conhecimento a respeito dessa população.

Universidade Estadual do Sudoeste da Bahia, Departamento de Saúde, Curso de Fisioteraia. Jequié, BA, Brasil

2 Universidade Estadual do Sudoeste da Bahia, Departamento de Saúde, Programa de Pós-Graduação em Ciências da Saúde. Jequié, BA, Brasil

3 Universidade Estadual do Sudoeste da Bahia, Departamento de Saúde, Curso de Enfermagem. Jequié, BA, Brasil

4 Faculdade de Tecnologia e Ciências. Curso de Enfermagem. Jequié, BA, Brasil.

5 Universidade Federal do Rio Grande do Norte, Centro de Ciências da Saúde, Departamento de Enfermagem. Natal, RN, Brasil.
Palavras-chave:

Envelhecimento da População.

Indicadores Básicos de Saúde. Taxa de Fecundidade.

Mortalidade. Saúde Pública. Condições Sociais. Análise

Quantitativa.

Idoso. Jequié-BA.

Correspondência / Correspondence

Luciana Araújo dos Reis

Rua Magno Senhorinho, 265. Jequiezinho

45206-179 Jequié, BA, Brasil

E-mail: cianareis@hotmail.com 


\section{Abstract}

The population's aging is a response to the change of some health indicators, especially the decrease in fecundity and mortality and increase in life expectation. The population growth in Brazil is a matter of public health, and it has been increasing a lot over the last decades. Therefore, this study aims to identify health and socio-demographic conditions of elderly living at home in the city of Jequié-BA. It's a descriptive, cross-sectional study with a qualitative approach. It was identified that $51.7 \%$ of the aged were male, $37.8 \%$ belonged to the age group of $70-79$ years and $96.5 \%$ were illiterate; the most frequent pathology among them was hypertension, with $73.3 \%$, followed by diabetes mellitus, with $17,2 \%$. The study reinforces the importance of planning public policies towards the specific needs of the elderly population,

Key words:

Demographic Aging.

Health Status

Indicators. Fecundity

Rate.Mortality. Public

Health. Social

Conditions.

Quantitative Analysis. Aged. Jequié city-BA. besides other studies that may enlarge the knowledge about this population.

\section{INTRODUÇÃO}

O envelhecimento populacional é uma resposta à mudança de alguns indicadores de saúde, especialmente a queda da fecundidade e da mortalidade e o aumento da expectativa de vida. Não é homogêneo para todos os seres humanos, sofrendo influência dos processos de discriminação e exclusão associados a gênero, etnia, racismo, condições sociais e econômicas, região geográfica de origem e localização de moradia. ${ }^{1} \mathrm{O}$ Brasil experimentou nas duas últimas décadas uma mudança na mortalidade proporcional, com queda na proporção para os menores de um ano e aumento da proporção de óbitos na faixa etária de idade de 50 anos ou mais. ${ }^{2}$

A longevidade é, sem dúvida, um triunfo. Há, no entanto, importantes diferenças entre os países desenvolvidos e os países em desenvolvimento. Enquanto nos primeiros o envelhecimento ocorreu associado às me- lhorias nas condições gerais de vida, nos outros esse processo acontece de forma rápida, sem tempo para uma reorganização social e da área de saúde adequada para atender às novas demandas emergentes. Para o ano de 2050, a expectativa no Brasil, bem como em todo o mundo, é de que existirão mais idosos que crianças abaixo de 15 anos, fenômeno nunca antes observado. ${ }^{1,3}$

No Brasil, em 1991 a população idosa era de aproximadamente 10,7 milhões de habitantes, mostrando, desde então, a importância deste contingente populacional. Atualmente estima-se que existam cerca de 17,6 milhões de idosos. ${ }^{2}$ No contexto de Jequié$\mathrm{BA}$, a população idosa representa 9,6\% da população total e, destes, $1,2 \%$ possui idade acima de 80 anos. $^{4}$

O crescimento elevado da população idosa brasileira é uma questão de saúde pública e vem acentuando-se muito nas últimas décadas. O país deixa de ser essencialmente jovem e passa a amadurecer. Essa transição 
demográfica vem acompanhada por um fenômeno chamado de transição epidemiológica, que é a mudança de uma população envelhecida, com baixa mortalidade, provocando profunda alteração no perfil da morbidade e causas de morte dessa população. Basicamente acontece a substituição das doenças infecciosas e parasitárias pelas doenças crônico-degenerativas. ${ }^{5}$

O envelhecimento pode ser compreendido como um processo natural, de diminuição progressiva da reserva funcional dos indivíduos - senescência - o que, em condições normais, não costuma provocar qualquer problema. No entanto, em condições de sobrecarga, como por exemplo, doenças, acidentes e estresse emocional, pode ocasionar uma condição patológica que requeira assistência - senilidade. Cabe ressaltar que certas alterações decorrentes do processo de senescência podem ter seus efeitos minimizados pela assimilação de um estilo de vida mais ativo. ${ }^{6}$

De acordo com os autores mencionados nesse estudo, a população idosa está em constante crescimento, sendo que as doenças que os acometem são influenciadas por diversos fatores. Deste modo, com o intuito de traçar uma melhor terapêutica e, consequentemente, proporcionar qualidade de vida aos idosos residentes em domićlío no município de Jequié-BA, é relevante para os profissionais de saúde conhecer as reais condições de saúde da população em estudo. Nesta perspectiva, este estudo objetiva conhecer as condições de saúde e sociodemográficas de idosos residentes em domicílio no município de Jequié-BA.

\section{METODOLOGIA}

Trata-se de uma pesquisa de caráter exploratório descritivo com delineamento transversal e abordagem quantitativa. O local de estudo foi o domić́lio de idosos residentes na área de abrangência da Unidade Básica de Saúde Padre Hilário, no Bairro do Inocoop, no município de Jequié-BA. A amostra foi composta por 29 idosos (idade maior ou igual a 60 anos) cadastrados ao Projeto de Extensão "Saúde do idoso na família, asilo e na comunidade: vivenciando rede suporte social no cuidado ao idoso", cadastrado na Universidade Estadual do Sudoeste da Bahia (UESB). O local de estudo foi o Bairro do Inocoop, pelo fato de o projeto citado acima desenvolver suas atividades nesse bairro e por este servir como campo de estágio para os cursos de Enfermagem e Fisioterapia da UESB. Como instrumento para a coleta de dados gerais dos idosos, utilizou-se uma ficha adaptada para as informações de interesse neste estudo, contendo dados sociodemográficos e de saúde.

Os procedimentos foram realizados após parecer favorável do Comitê de Ética da UESB, atendendo aos aspectos éticos constantes na Resolução no 196/96 do Conselho Nacional de Saúde. Para participar da pesquisa, o idoso ou seu responsável assinou um Termo de Consentimento Livre e Esclarecido, podendo o idoso desistir do estudo em qualquer fase de sua realização. ${ }^{7}$

A coleta de dados foi realizada após parecer favorável do Comitê de Ética e Pesquisa (CEP) da UESB. Foi encaminhada a Unida- 
de Básica de Saúde uma carta de apresentação e, após a liberação da realização do estudo, os agentes de saúde serviram como elo entre a pesquisadora e os idosos. A coleta de dados foi realizada pelos autores da pesquisa em encontros pré-agendados com os idosos, respeitando-se suas atividades cotidianas.

Os dados foram organizados e tabulados em banco de dados gerado pelo software Microsoft Excel, no qual foi realizado o tratamento estatístico descritivo.

\section{RESULTADOS}

A partir da análise do questionário utilizado na pesquisa, pode-se observar que $51,7 \%$ dos idosos eram do sexo masculino, $37,8 \%$ possuíam idade entre a faixa etária de 70 a 79 anos e 96,5\% não eram alfabetizados. Destaca-se também que $82,7 \%$ eram aposentados, sendo que $86,2 \%$ possuíam renda de até um salário mínimo, como demonstra a tabela 01 .

Tabela 1 - Distribuição dos idosos de acordo com as variáveis sociodemográficas. JequiéBA, 2008.

\begin{tabular}{lcc}
\hline Variáveis & N & $\%$ \\
\hline Sexo & 15 & 51,7 \\
$\quad$ Masculino & 14 & 48,3 \\
$\quad$ Feminino & & \\
Idade & 10 & 34,5 \\
$\quad 60$ - 69 Anos & 11 & 37,8 \\
70 - 79 Anos & 8 & 27,6 \\
$\quad 80$ Anos & & \\
Escolaridade & 28 & 96,5 \\
$\quad$ Não Alfabetizado & 1 & 3,5 \\
Alfabetizado & & 82,7 \\
Profissão & 24 & 13,8 \\
Aposentado & 4 & 3,5 \\
Do Lar & 1 & 86,2 \\
Feirante & & 13,8 \\
Renda & 25 & \\
Até 1 salário mínimo & 4 & \\
Não Possui & & \\
\hline
\end{tabular}


De acordo com o diagnóstico clínico, evidenciou-se que a patologia mais encon- trada entre os idosos foi a hipertensão, com $73,3 \%$ dos acometimentos, seguida do diabetes, com $17,2 \%$ (tabela 02 ).

Tabela 2 - Distribuição dos idosos quanto ao diagnóstico clínico. Jequié-BA, 2008.

\begin{tabular}{lcc}
\hline \multicolumn{1}{c}{ Patologias } & N & $\%$ \\
\hline Hipertensão & 23 & 73,3 \\
Diabetes & 5 & 17,2 \\
Hipercolesterolemia & 1 & 3,4 \\
Osteoporose & 1 & 3,4 \\
Déficit Visual & 2 & 6,8 \\
AVE & 3 & 10,3 \\
\hline
\end{tabular}

$\mathrm{Na}$ tabela 03, verificou-se a distribuição dos idosos em relação à dependência, sendo $24,1 \%$ dos idosos classificados como dependentes para a realização das atividades básicas e instrumentais de vida diária, apesar da maioria, $75,9 \%$, relatar independência para realizá-las. Destaca-se ainda que, entre os idosos, nenhum se encontrava acamado, portanto $100 \%$ dos idosos possuíam ao menos algum grau de mobilidade no domićlíio.

Tabela 3 - Distribuição dos idosos quanto à independência das atividades da vida diária. Jequié-BA, 2008.

\begin{tabular}{lcc}
\hline \multicolumn{1}{c}{ Dependente } & $\mathrm{N}$ & $\%$ \\
\hline Sim & 7 & 24,1 \\
Não & 22 & 75,9 \\
Total & 29 & 100 \\
\hline
\end{tabular}

\section{DISCUSSÃO}

Após a coleta de dados dos idosos, através da ficha adaptada, foi possível adquirir informações para análise desta pesquisa. Mais da metade da amostra era composta por ho- mens $(51,7 \%)$, embora no Brasil o número absoluto de mulheres idosas seja superior quando confrontado com o de homens acima de 65 anos. Isso pode acontecer pela existência da mortalidade diferencial entre os sexos, inclusive na população brasileira. ${ }^{8}$ 
Segundo dados da Pesquisa Nacional por Amostra de Domicílios, a maior proporção de idosos na população brasileira tem entre 60-69 anos, mas a proporção de idosos com 70 anos ou mais tem aumentado. Os idosos com 70-79 anos representam $37,8 \%$ da população deste estudo. ${ }^{9}$ Dessa forma, os idosos necessitarão de cuidados mais específicos, sendo necessária a capacitação dos profissionais de saúde no seu cuidado e adequação dos serviços de saúde para atender satisfatoriamente a esse contingente populacional.

O baixo nível de escolaridade da maioria dos idosos do presente estudo (96,5\% de analfabetos) confirma o encontrado em outros estudos desenvolvidos com este mesmo grupo etário. Considera-se que, possivelmente, essa baixa escolaridade dos idosos seja reflexo da taxa de alfabetização nos anos 20-40 do século passado, quando não havia tanta cobrança por nível escolar como atualmente e o papel das mulheres na sociedade era casar, ter filhos e cuidar de casa e, via de regra, não eram estimuladas a estudar. ${ }^{10}$

O estudo detectou alta prevalência de aposentados $(82,7 \%)$, sendo que a maioria destes, 86,2\%, possuía renda de até um salário mínimo. Estes dados refletem a realidade de Jequié, pois, segundo o último censo do IBGE, realizado em 2000, 34.105 habitantes do referido município, que representam 23,17\% da população total, possuem esta renda. ${ }^{11}$ Observa-se também que a maioria desses idosos tem dependentes e suas aposentadorias constituem importante fonte de renda, ou mesmo a principal renda da família. ${ }^{12}$
Neste estudo pôde-se ainda observar uma proporção elevada de portadores de doenças crônico-degenerativas, fenômeno este que pode ser explicado pela transição epidemiológica. ${ }^{5} \mathrm{O}$ acometimento mais comum foi hipertensão arterial, com 73,3\%. Segundo o VI Relatório do Joint National Committee, estudos populacionais indicam que a prevalência da hipertensão arterial aumenta de acordo com a elevação da faixa etária, o que sugere a ênfase na prevenção primária e na ampliação do diagnóstico precoce, sugerindo novas formas de atuação que se baseiem no processo educacional e de conscientização capazes de alcançar melhores resultados a curto, médio e longo prazos. ${ }^{13}$

Diferentemente da hipertensão, a proporção de diabetes, embora considerável, foi menor, com $17,2 \%$. No estudo multicêntrico sobre prevalência do diabetes mellitus realizado no Brasil, verificou-se que o aumento de prevalência do diabetes ocorre proporcionalmente à idade. Os autores deste estudo alertam para a importância dessa enfermidade, em razão do envelhecimento populacional que vem ocorrendo no país, reforçando que essa constatação corrobora estudos realizados em países desenvolvidos e em desenvolvimento. ${ }^{14}$

Quanto à relação de dependência, 24,1\% dos idosos investigados referiram necessitar de ajuda para as atividades de vida diária (AVD). O processo de envelhecimento traz consigo limitações que muitas vezes fragilizam a capacidade funcional dos idosos como tontura, instabilidade, diminuição da 
acuidade auditivo e visual, entre outros - e os leva à dependência, geralmente significando necessidade de cuidados permanentes. ${ }^{4}$ Portanto, torna-se importante considerar o nível de funcionalidade desses idosos e estimulá-los a manter o máximo de independência possível que eles consigam alcançar dentro de suas limitações.

\section{CONCLUSÃO}

O estudo revela a necessidade de um enfoque mais amplo dos fatores que proporcionam um envelhecimento saudável, pois este é resultado da interação multidimensional entre a saúde física e mental, au-

\section{REFERÊNCIAS}

1. Pereira RS, Curioni CC, Veras R. Perfil demográfico da população idosa no Brasil e no Rio de Janeiro em 2002. Textos sobre Envelhecimento 2003; 6(1): 43-59.

2. Coelho Filho JMC, Ramos LR. Epidemiologia do envelhecimento no Nordeste do Brasil: resultados de inquérito domiciliar. Rev Saúde Pública 1999; 33(5): 445-53.

3. Garcia RA, Carvalho JAM. O envelhecimento da população brasileira: um enfoque demográfico. Cad Saúde Pública 2003; 19(3): 725-33.

4. Chaimowiicz F. A saúde dos idosos brasileiros às vésperas do século XXI: problemas, projeções e alternativas. Rev Saúde Pública 1997; 31: 184-200. tonomia, integração social, suporte familiar e independência econômica.

Segundo Veras \& Camargo, ${ }^{15}$ para garantir melhoria na qualidade de vida dos idosos, é preciso enfrentar um duplo desafio: assegurar serviços de qualidade para este segmento e desenvolver recursos humanos de excelência e conhecimento para lidar com o grupo etário que mais cresce em nosso país.

Diante disso, observa-se a importância do planejamento de políticas públicas direcionadas às necessidades específicas da população idosa, além de outros estudos que permitam ampliar o conhecimento a respeito dessa população.

5. Lima-Costa MF, Veras R. Saúde pública e envelhecimento, Rio de Janeiro. Cad Saúde Pública 2007; 19(3): 15-21.

6. Ramos LR. Determinant factors for healthy aging among senior citizens in a large city: the Epidoso Project in São Paulo. Cad Saúde Pública 2003; 19(3): 8-14.

7. Brasil. Ministério da Saúde. Conselho Nacional de Saúde. Resolução no 196/96 sobre pesquisa envolvendo seres humanos. Brasília: Ministério da Saúde, 1996. 24 p.

8. Laurenti R, Lebrão ML. Saúde, bem-estar e envelhecimento: o estudo SABE no município de São Paulo. Revista brasileira de epidemiologia 2005; 8(2): 127-41. 
9. Instituto Brasileiro de Geografia e Estatística. Pesquisa Nacional de Amostra de Domicilio PNAD. Rio de Janeiro: IBGE; 2000.

10. Menezes TN, Lopes FJM, Marucci MFN. Estudo domiciliar da população idosa de Fortaleza/CE: aspectos metodológicos e características sóciodemográficas. Revista brasileira de epidemiologia 2007;10(2): 168-77.

11. Instituto Brasileiro de Geografia e Estatística. Censo 2000. Rio de Janeiro: IBGE; 2002.

12. Machado JC, et al. Avaliação do declínio cognitivo e sua relação com as características socioeconômicas dos idosos em Viçosa-MG. Revista brasileira de epidemiologia 2007; 10(4): 592-605.
13. Joint National Committee. The sixth report of the IV Joint National Committee on prevention detection evaluation and treatment of high blood pressure. Archive International Medicine 1997; 157: 2413-46.

14. CENEPI. Estudo multicêntrico sobre a prevalência do diabetes mellitus no Brasil. Informe epidemiológico do SUS 1992; 1(3): 45-73.

15. Veras RP, Camargo Jr, KR. Idosos e universidade: parceria para qualidade vida. In. Veras RP, organizador. Terceira idade: um envelhecimento digno para o cidadão do futuro. Rio de Janeiro: Relume-Dumará, UnATI; 1995. p.11-27.

Recebido: 20/2/2008

Revisado: 5/8/2008

Aprovado: 2/5/2009 